\title{
ENSAYO BIOGEOGRAFICO SOBRE LA PTERIDOFLORA DE SIERRA NEVADA (GRANADA, ESPAÑA)
}

\author{
JUAN VARO (*) \& A. ENRIQUE SALVO (**)
}

\section{RESUMEN}

Se realiza un estudio corológico y ecológico de los pteridófitos de Sierra Nevada. Se hace una valoración del origen de la pteridoflora de dicha Sierra.

\section{SUMMARY}

A chorological and ecological study of the pteridophytes located in the Sierra Nevada (Spain) was carried out. An evaluation on the origin of these species in the above mentioned mountain is investigated.

\section{INTRODUCCION}

Dentro de la Cordillera Bética, el macizo nevadense destaca por su elevada altitud alcanzando las cotas más elevadas de la Península Ibérica (Mulhacen 3.482 m). Esta montaña, relativamente joven ( 20 millones de años), presenta una alta variabilidad petrológica. Las rocas mejor representadas son por una parte los esquistos y por otra las calizas y dolomías. Son precisamente los primeros quienes predominan en Sierra Nevada, formando casi la totalidad de la "zona central", las calizas y dolomías, sin embargo, abundan en la "zona periférica”. Es preciso destacar que estas dos zonas no están en contacto directo, sino que entre ambas se ha puesto de manifiesto la existencia de una serie especial de pizarras metamórficas, bastante enriquecidas en mármoles, conocida como Mischungzone.

No es hasta el período Mioceno cuando los materiales nevadenses van a formar un pliegue anticlinal. Este plegamiento es progresivo y se continúa durante el Plioceno, siendo entonces cuando Sierra Nevada se desarrolla como conjunto montañoso netamente individualizado, acentuándose su relieve y alcanzando cada vez mayores latitudes.

Comunicación presentada al III Simposio Nacional de Botánica Criptogámica. Málaga, 1978

i*) Departamento de Botanica, Facultad de Ciencias, Universidad de Granidat.

(**) Departamento de Botánica, Facultad de Ciencias, Universidad de Málaga. 
Los suelos que se producen a partir de estas rocas son de distintos tipos, así cabe destacar sobre rocas silíceas: protoranker, ranker mulliforme alpino, tierras pardas oligotrófilas y suelos orgánicos (Anmoor); y sobre roca caliza: Litosoles, rendsinas y suelos pardos calizos.

En Sierra Nevada pueden observarse todos los pisos bioclimáticos definidos para la región mediterránea por Rivas-Martínez (1981), a excepción del piso termomediterráneo. Al piso mesomediterráneo en Sierra Nevada le corresponde generalmente un dominio climácico de Paeonio-Quercetum rotundifoliae, encinares frios desarrollados sobre suelos calizos. Esta formación en la vertiente sur, donde asciende hasta los $1.600 \mathrm{~m}$, se enriquece en elementos termófilos del Oleo-Quercion rotundifolio-suberis. El dominio climácico del supramediterráneo nevadense esta representado por los acerales y serbales (Daphno-Aceretum granatense), que encuentran su óptimos en los barrancos y lugares protegidos de suelos calizos, frescos y profundos, mientras que sobre suelos silíceos serán los bosques de robles (Quercetum pyrenaicae nevadense) la vegetación climácica en este piso. A partir de los 1.600 $\mathrm{m}$, sobre suelo calizo, los pinares con enebros y sabinas (Daphno oleoidi-Pinetum sylvestris), constituyen el dominio climácico del piso oromediterráneo, mientras que sobre sílice se ve sustituido por un enebral carente de estrato superior (Genisto beticae-Juniperetum hemisphericae). La vegetación potencial del piso crioromediterráneo, por encima de los $2.600 \mathrm{~m}$, está constituida por hemicriptófitos y caméfitos del Festucetum clementei. (Rivas Goday \& Rivas-Martínez, 1971; Rivas-Martínez \& al., 1977; Rivas-Martínez, 1981).

Corológicamente Sierra Nevada se encuentra dentro de la provincia Bética, constituyendo en su mayor parte el sector nevadense (Rivas Martínez \& al., 1977). Este sector se caracteriza por poseer endémica un 36\% de su flora (Favarger, 1972), siendo a partir de los 2.800 m donde alcanza su tasa más elevada, 75\% (Gómez Campo, 1974). Este porcentaje resulta ser el más alto de la Península Ibérica, y uno de los más altos de Europa.

\section{COMPOSICION PTERIDOFLORISTICA DE SIERRA NEVADA}

El estudio realizado por nosotros pone de manifiesto la existencia en Sierra Nevada de 46 táxones de pteridófitos (40 especies, 2 subespecie y 4 híbridos) que a continuación se detallan.

Cada táxon va seguido de una serie de connotaciones ecológicas cuyo código es el siguiente:

a) Frecuencia: los índices utilizados son los de Willmanns \& Rasbach (1973):

vr: muy raro (sólo 1 a 3 localidades de máximo $100 \mathrm{~m}^{2}$ ); r: raro; o: ocasional; c : común; vc: muy común.

b) Altitud: Hemos preferido utilizar los pisos bioclimáticos de RivasMartínez (1981):

M: mesomediterráneo; S: Supramediterráneo; O: oromediterráneo; C: crioromediterráneo; ( ): raro en ese piso.

c) Comportamiento ecológico:

Ru: rupícola; Te: terrícola; G: glerícola; Ri: ripícola; Hu: humícola; Es: esciófilo (caso extremo); Hi: higrófilo (caso extremo)- (Si): Silicícola; (Ca): Calcícola. 
Selaginella denticulata (L.) Link.

Isoetes velata Braun

Equisetum telmateia Ehrh.

Equisetum arvense L.

Equisetum ramosissimum Desf.

Equisetum x moorei Newman

Equisetum variegatum Schleicher

Botrychium lunaria (L.) Swartz

Ophioglossum lusitanicum L.

Ophioglossum vulgatum $\mathrm{L}$.

Polypodium vulgare $\mathrm{L}$.

Polypodium cambricum L. subsp. australe

(Fée) Brudet \& Greuter

Polypodium x fontqueri Rothm.

Cheilanthes vellea (Ait.) Muell

Cheilanthes pteridioides (Reichard) C.Chr.

Cheilanthes guanchica Bolle

Cheilanthes maderensis Lowe

Cryptogramma crispa (L.) R.Br.

Pteris vittata L.

Adiantum capillus-veneris $\mathrm{L}$.

Pteridium aquilinum (L.) Kuhn

Anogramma leptophylla (L.) Kuhn

Asplenium petrarchae (Guerin) DC. subsp. petrarchae

Asplenium trichomanes L. subsp. pachyrachys (Christ)

Lowis \& Reichstein

Asplenium trichomanes L. subsp. quadrivalens Meyer

Asplenium viride $\mathrm{Hudson}$

Asplenium foresiacum (Le Grand) Christ

Asplenium $\mathrm{x}$ costei Litard.

Asplenium adiantum-nigrum $\mathrm{L}$.

Asplenium onopteris L.

Asplenium septentrionale (L.) Hoffm.

Asplenium ruta-muraria L. subsp. ruta-muraria

Asplenium ceterach L.

Asplenium scolopendrium $\mathrm{L}$.

Asplenium subglandulosum (Hook. et Grev.) Salvo, Prada

\& Diaz subsp. hispanicum (Cosson)Salvo,

Prada \& Diaz

Athyrium filix-femina (L.) Roth

Cystopteris fragilis (L.) Bernh.

Cystopteris dickieana R. Sim.

Dryopteris filix-mas (L.) Schott

Dryopteris affinis (Lowe) Fraser-Jenkins subsp. borreri

(Newman) Frasser-Jenkins

Dryopteris affinis (Lowe) Fraser-Jenkins subsp. affinis

Dryopteris submontana (Fraser-Jenkins \& Jermy)

Fraser-Jenkins
r/M/TeHi

vr/M/TeHi

c/MS/Ri

c/MS/Ri

c/M/Te

$\mathrm{vr} / \mathrm{M} / \mathrm{Te}$

$\mathrm{vr} / \mathrm{M} / \mathrm{Hi}$

$\mathrm{r} / \mathrm{O} / \mathrm{Hi}$

$\mathrm{r} / \mathrm{M} / \mathrm{Hi}$

$\mathrm{r} / \mathrm{S} / \mathrm{Hi}$

o/OC/RuEs(Si)

o/M(S)/Ru(Ca)

$\mathrm{vr} / \mathrm{S} / \mathrm{Ru}$

$\mathrm{r} / \mathrm{M} / \mathrm{Ru}(\mathrm{Ca})$

c/MS/RuTe(Ca)

vr/M/TeEs

$\mathrm{r} / \mathrm{M} / \mathrm{RuEs}$

o/OC/G

o/M/RuHi (Ca)

vc/MS/RuHi (Ca)

$\mathrm{c} / \mathrm{MS} / \mathrm{Te}(\mathrm{Si})$

o/MS/Te

$\mathrm{c} / \mathrm{MS} / \mathrm{Ru}(\mathrm{Ca})$

r/OC/Ru(Si)

vc/MS/Ru(Ca)

$\mathrm{o} / \mathrm{OC} / \mathrm{Ru}$

r/S/TeHi(Si)

vr/S/TeHi(Si)

o/OC/Ru(Si)

c/MS/Ru

o/OC/Ru(Si)

c/MS(o)/Ru

vs/MSOC/Ru

r/MS/TeHi(Ca)

r/MS/Ru(Ca)

vc/MS/RiHu

$\mathrm{vc} / \mathrm{MSOC} / \mathrm{Ru}$

o/O/Ru

vc/MSO/TeHu

o/SO/Te

r/S/Te

r/SO/Te(Ca) 
Dryopteris tyrrhena Fraser-Jenkins \& Reichstein

$\mathrm{r} / \mathrm{SO} / \mathrm{Te}(\mathrm{Si})$

Pobystichum lonchitis (L.) Roth

$\mathrm{o} / \mathrm{OC} / \mathrm{Ru}(\mathrm{Si})$

Polystichum acule atum (L.) Roth

o/S/TeHu

Pobystichum x illiricum (Borbás) Hahre

$\mathrm{r} / \mathrm{O} / \mathrm{Ru}$

\section{COMENTARIOS ECOLOGICOS}

Sobre los $2.400 \mathrm{~m}$, en las proximidades de lagunas y hondonadas, debido a la fusión de las nieves en verano se forman unas praderas subhigrófilas (Nardo-Festucetum violaceae) pertenecientes a la clase Nardetea. Especie característica de esta clase es precisamente Botrychium lunaria. Este taxon cosmopolita se presenta en el macizo nevadense con un gran aislamiento, debido al retroceso de las glaciaciones y ubicación de esta especie circumboreal en las grandes altitudes. En comunidades de pastizales permanentemente encharcadas (Molinio-Arrbenateretea), en el piso supramediterráneo, aparece otro helecho eusporangiado, Ophioglossum vulgatum. La otra especie de este género aquí representada, O. lusitanicum, aparece en comunidades semejantes pero alt itudinalmente más bajas, en el piso mesomediterráneo. Por su parte Isoetes velata, también de comunidades higrófilas, prefiere los pastizales terofíticos encharcados temporalmente en invierno (Isoetion).

En el Aro-Ulmetum, comunidades riparias del mesomediterráneo, se refugían una serie de pteridófitos que alcanzan su óptimo en los bosques frescos más septentrionales: Equisetum telmateia, Athyrium filix-femina, Dryopteris filix-mas, además de $P$. acule atum. Equisetum telmateia es sustituído en aquellas estaciones más nitrificadas o bien donde la materia orgánica se descompone difícilmente por $E$. arvense. Equisetum variegatum prefiere las situaciones totalmente sumergidas, en los cursos de aguas calizas de fuerte escorrentía. Dentro de esta comunides de Aro-Ulmetum cuando las condiciones microclimáticas padecen cierta atlanticidad hemos podido observar Dryopteris affinis subsp. affinis.

Muy pobres son aquellas comunidades de paredes calizas rezumantes de la clase Adiantetea, de la que es especie característica Adiantum capillus-veneris. En la vertiente Sur, dentro del piso mesomediterráneo, en aquellas estaciones más térmicas y con un protosuelo más desarrollado, Pteris vittata, elemento pantropical distribuido en el mediterráneo occidental, aparece en este tipo de comunidades de Adiantete.a. Asplenium scolopendrium comparte este tipo de ecología, si bien prefiere aquellos nichos más acidificados. Cuando el $\mathrm{pH}$ desciende por debajo de la neutralidad en determinadas estaciones aparece Asplenium foresiacum. Anogramma leptophylla coloniza aquellos sustratos rezumantes o donde la humedad edáfica es elevada, muy desarrollados y de $\mathrm{pH}$ ácido. Todos estos elementos raramente superan el supramediterráneo.

Aproximadamente la mitad de los helechos que crecen en Sierra Nevada prefiere colonizar los biótopos rupícolas (Asplenietea rupestria). Es evidente que uno de los factores más importantes que van a determinar el tipo de comunidad que va a asentarse sobre un sustrato rocoso es la naturaleza de ese sustrato. Así, existe una gran diferencia entre las comunidades rupícolas calcófilas y silicófilas, siendo generalmente más ricas las primeras que las segundas. En Sie rra Nevada está separación se nos presenta altitudinalmente, de tal forma que al ser caliza la zona periférica, 
por tanto más baja, y de sílice la zona central, más alta, en el primer tipo de sustra. to van a abundar los táxones mediterráneos (Asplenium onopteris, A. petrarchae subsp. petrarchae, A. trichomanes sbusp. quadrivalens, Cheilanthes pteridioides, Polypodium cambricum) y en el segundo los circumboreales (Asplenium adiantum-nigrum, $A$. trichomanes subsp. pachyrachys, $A$. viride, $A$. septentrionale, Pobpodium vulgare). Además en estas comunidades aparecen una serie de pteridófitos ubiquistas: $A$ splenium ceterach, Asplenium ruta-muraria y Cystopteris fragilis. Numerosas situaciones microclimáticas pueden modificar esta composición general. Así, cuando las rocas calizas se encuentran dolomitizadas suele aparecer Asplenium subglandulosum subsp. hispanicum. En el piso supramediterráneo, en su horizonte superior, dentro de las poblaciones de Pobpodium cambricum, aparece ocasionalmente el híbrido triploide $P$. $x$ fontqueri. El género Cheilanthes en Sierra Nevada ofrece un ejemplo revelador de desplazamientos por variaciones estacionales, debidas tanto a la exposición lumínica, y por tanto térmica, como de la naturaleza del sustrato. De esta forma, $C$. velle a coloniza aquellos hábitats expuestos, $C$. maderensis desplaza a C. pteridioides en aquellos otros más resguardados y $C$. guanchica cuando el sustrato ha sufrido cierta acidificación. Mezclado en las poblaciones de Cystopteris fragilis es frecuente encontrar el taxon de esporótipo rugoso, $C$. dickieana.

Son también comunidades ricas en elementos alpin os aquellos quionófilas de pedregales sueltos del Dryopteridion oreadis (Cryptogrammo-Dryopteridetum oreadis). De óptimo eurosiberiano, a parece en el piso oro y crioromediterráneo de Sierra Nevada muy desdibujada. Polystichum lonchitis y Cryptogramma crispa, junto con Dryopteris tyrrhena, elemento mediterráneo que sustituye a $D$. oreades, son los constituyentes pteridofíticos de esta comunidad. Por otra parte, en el supramediterráneo y sobre sustrato calizo es frecuente encontrar en Sierra Nevada una comunidad similar a la anterior, formada por Dryopteris submontana.

\section{COMENTARIOS BIOGEOGRAFICOS}

A continuación se distribuyen los táxones de pteridófitos que crecen en Sierra Nevada en los grupos corológicos establecidos por Pichi-Sermolli (1979) para los pteridófitos de la Región Mediterránea.

A. Especies que colonizaron el Mediterráneo antes o durante el Terciario.

1. Especies de amplia distribución en el mundo (Cosmopolitas).

Anogramma leptophylla

Adiantum capillus-veneris

Asplenium trichomanes s.l.

Equisetum ramosissimum

Ophioglossum vulgatum

Pteridium aquilinum

2. Especies de amplia distribución en el hemisferio norte (Circumboreales).

Cystopteris dickieana

Equisetum telmateia

3. Especies de areal mediterráneo ampliado hacia el este.

Asplenium ceterach

Cheilanthes vellea

Cheilanthes pteridioides

Ophioglossum lusitanicum

4. Especies mediterráneas y de regiones adyacentes (Submediterráneas). Asplenium scolopendrium

Polypodium cambricum

Selaginella denticulata 
5. Especies ampliamente distribuidas en trópicos y subtrópicos (Pantropicales).

Pteris vittata

6. Relictos paleomediterráneos (no existen).

7. Especies mediterráneas.
Asplenium onopteris
Cheilanthes maderensis
Asplenium petrarchae
Dryopteris submontana
Cheilanthes guanchica
Isoetes veiata

8. Especies distribuidas en Región Mediterránea y Saharo-Arábiga (no existen).

9. Relíctos Ilírico-Panóricos (no existen).

10. Endemismos mediterráneos.

Asplenium subglandulosum

subsp. hispanicum

Dryopteris tyrrhena

B. Especies que colonizaron el Mediterráneo durante el cuaternario.

1. Especies de amplia distribución en el mundo (Cosmopolitas). Botrychium lunaria Cystopteris fragilis

2. Especies ampliamente distribuídas en el hemisferio norte (Circumboreales).

Asplenium adiantum-nigrum

Asplenium ruta-muraria

Asplenium septentrionale

Dryopteris filix-mas

Asplenium viride

Equisetum arre'nse

Athyrium filix-femina

Equisetum variegatum

Cryptogramma crispa

Polypodium vulgare

Polystichum lonchitis

3. Especies cuya distribución esta centrada en la región eurosiberiana. Asplenium foresiacum

Dryopteris affinis s.l.

Polystichum acule atum

En la siguiente tabla se presentan los porcentajes de cada grupo en Sierra Nevada, comparándose con el conjunto de la pteridoflora mediterránea, y con aquellos resultados obtenidos para las sierras de Algeciras (Diez Garretas \& Salvo, 1980). Estas sierras béticas, separadas escasamente por $300 \mathrm{~km}$, presentan una flora y vegetación epiontológicamente diferentes. 


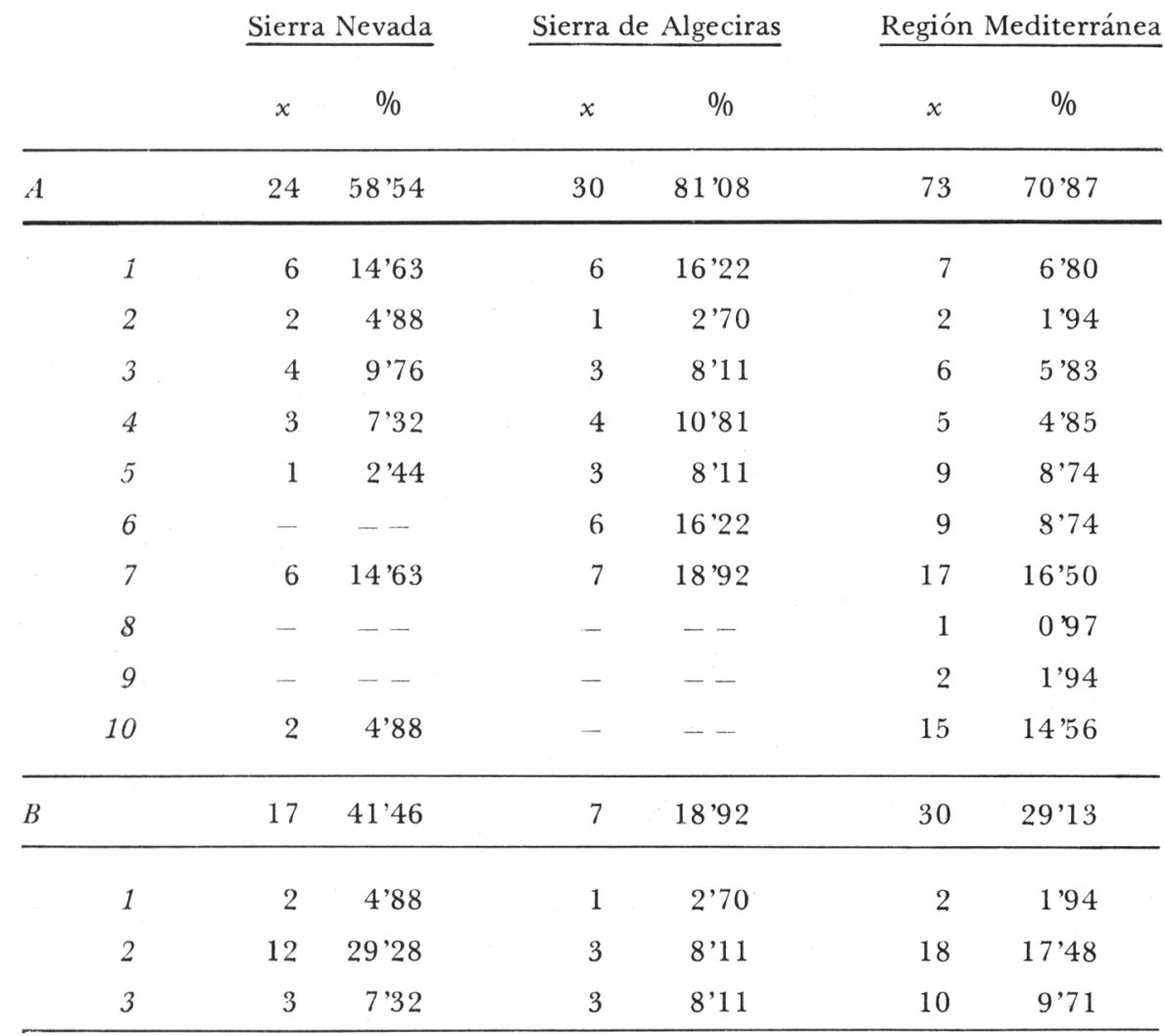

De los resultados obtenidos deducimos las siguientes conclusiones:

1. En Sierra Nevada existe un total de 40 especies de pteridófitos, lo cual representa un 38 ' $83 \%$ de la pteridoflora mediterránea.

2. La Sierra de Algeciras y Sie rra Nevada, separadas escasamente por $300 \mathrm{~km}$, albergan un total de 60 especies, lo que supone que estas sierras béticas albergan un $60 \%$ de los helechos que crecen en el Mediterráneo.

3. Es notable el elevado porcentaje (41'46\%) que presenta en Sierra Nevada el grupo de táxones que colonizaron la región mediterránea durante el cuaternario. Este índice se debe fundamentalmente al elevado número de especies circumboreales, $(2922 \%)$, la mayoría de los cuales encontraron en los pisos oro y crioromediterráneo del macizo nevadense un refugio durante la retirada de las glaciaciones. Este evento climatológico no influenció apenas a la Sie rra de Algeciras, por ello los índices del grupo $\mathrm{B}$ son tan dispares entre ambas sierras.

4. La conclusión anterior se ve aún más apoyada por el hecho de que en Sierra Nevada no existen relictos paleomediterráneos, mientras que en la Sierra de Algeciras es uno de los mejor representados (16'22\%). Al igual ocurre con el grupo de especies pantropicales (A.5) cuyo índice es muy inferior al de la Región Mediterránea y Sierra de Algeciras. 
5. Es necesario destacar que el grupo de táxones cosmopolitas que colonizaron la región meditērránea antes o durante el terciario presenta en Sierra Nevada un porcentaje que duplica al de la Región Mediterránea.

6. Si bien la tasa de endemismos fanerogámicos nevadenses es muy elevada, es contradictorio el bajo índice de endemismos pteridofíticos en comparación con el presentado por el total de la región mediterránea.

\section{BIBLIOGRAFIA}

DIEZ GARRETAS, B. \& SALVO, A.E. -1981- Ensayo biogeográfico de los pteridófitos de las Sierras de Algeciras. Anales Jard. Bot. Madrid 37 (2): 455-462.

FAVARGER, C. -1972- Endemism in the Montane Floras of Europe. In Ed. D.H. Valentine: Taxonomy, phytogeography and evolution. Academic Press. London.

GOMEZ CAMPO, C. -1974-Nuestra riqueza florística: orígenes y significado. Adena 48: 23-28.

PICHI-SERMOLLI, R. -1979- A survery of the pteridological flora of the Mediterranean Region. Webbia 34: 175-242. Firenze.

PIGNATTI, S. -1980- Flora d'Italia. Calderini. Bologna.

RIVAS GODAY, S.\& RIVAS MARTINEZ, S. - 1971-Vegetación potencial de la provincia de Granada. Trab. Dep. Botánica y F. Veg. 4: 3-85. Madrid.

RIVAS MARTINEZ, S. - 1981- Les étages bioclimatiques de la végétation de la Péninsule Ibérique. Anales Jard. Bot. Madrid 37 (2): 261-268.

RIVAS MARTINEZ, S., ARNAI, C. BARRENO, E. \& CRESPO, A. -1977- Apuntes sobre las provincias corológicas de la Península Ibérica e Islas Canarias. Opuscula Botánica Pharmaciae Complutensis 1: 148. Madrid.

WILLMANNS, O. \& RASBACH, H. -1973-Observations on the pteridophytes of São Miguel, Açores. Fern Gaz. 10: 315-329. London. 